\title{
Big bang and big crunch in matrix string theory
}

\author{
J. Bedford, ${ }^{1,2, *}$ C. Papageorgakis, ${ }^{3, \dagger}$ D. Rodríguez-Gómez, ${ }^{4, \$}$ and J. Ward ${ }^{1,2, \S}$ \\ ${ }^{1}$ Centre for Research in String Theory, Department of Physics, Queen Mary, University of London, \\ Mile End Road, London E1 4NS, United Kingdom \\ ${ }^{2}$ Department of Physics, CERN-Theory Division, 1211 Geneva 23, Switzerland \\ ${ }^{3}$ Department of Theoretical Physics, Tata Institute of Fundamental Research, Homi Bhabha Road, Mumbai 400 005, India \\ ${ }^{4}$ Joseph Henry Laboratories, Princeton University, New Jersey 08544, USA \\ (Received 20 February 2007; published 20 April 2007)
}

\begin{abstract}
Following the holographic description of linear dilaton null cosmologies with a big bang in terms of matrix string theory put forward by Craps, Sethi, and Verlinde, we propose an extended background describing a universe including both big bang and big crunch singularities. This belongs to a class of exact string backgrounds and is perturbative in the string coupling far away from the singularities, both of which can be resolved using matrix string theory. We provide a simple theory capable of describing the complete evolution of this closed universe.
\end{abstract}

DOI: 10.1103/PhysRevD.75.085014

PACS numbers: 04.65.+e, 98.80.Cq, 04.50.+h

\section{INTRODUCTION}

It is widely believed that string/M-theory should be singularity free in its moduli space. So far, the theory has succeeded in resolving a number of static singular regions, but null and spacelike singularities still pose a fundamental challenge. The standard cosmological paradigm assumes the existence of a big bang, out of which the Universe originated. Indeed, its relic signature is the cosmic microwave background radiation at a temperature of close to $2.7 \mathrm{~K}$ which we can measure today. Hence, both from the purely theoretical and the experimental points of view, the question of what happened at the big bang is of extreme interest and any progress in the understanding of nonstatic singularities is highly desirable.

An interesting step in this direction arising from string/ M-theory is a model proposed in [1]. By choosing a particularly simple background, namely, a lightlike linear dilaton (LLD), Craps et al. were able to obtain a matrix string theory description [2-5] of the early-time physics, which seems to be valid even at the big bang-type singularity. One of the main attributes of the LLD background is that the string world sheet theory is still free, since the dilaton does not contribute to the central charge of the conformal field theory (CFT). Therefore one would naively expect that it should be possible to make use of matrix string theory, by simply exchanging $g_{s}$ for $g_{s}\left(x^{+}\right)$. Indeed, the authors of [1] showed how this guess turns out to be correct and obtained a resolution of the singularity by explicitly deriving the matrix model via the discrete light-cone quantization (DLCQ) of string theory in the presence of an LLD and a sequence of dualities. Subsequently, the one-loop matrix potential was calculated

\footnotetext{
*Electronic address: j.a.p.bedford@qmul.ac.uk

${ }^{\dagger}$ Electronic address: costis@theory.tifr.res.in

Electronic address: drodrigu@ princeton.edu

${ }^{\S}$ Electronic address: j.ward@qmul.ac.uk
}

in [6] and was found to be attractive while vanishing at late times. ${ }^{1}$ These developments have generated great activity on closely related topics, e.g. [8-17]. Alternative holographic approaches to the study of nonstatic cosmological singularities involving the AdS/CFT correspondence have also been recently considered in the literature [18-23]. For a comprehensive list of references on both types of constructions as well as on the more general problem of resolving null and spacelike singularities in string/Mtheory, we refer the reader to [21] and the reviews [24,25].

In this note, starting with the LLD background, we will construct an extension to [1] containing a big bang and a big crunch while still having a perturbative matrix model description near the singularities. This is motivated by the analysis of the LLD cosmology: It is well known that the linear dilaton background can be obtained as a Penrose limit along a purely radially incoming/outgoing geodesic ${ }^{2}$ of an NS5 background (see for example [26]). This suggests a possible modification of the LLD background by taking the Penrose limit along an outgoing geodesic and gluing it with the Penrose limit along an incoming geodesic at the point $x^{+}=0$. The resulting background is simply flat space with a lightlike linear dilaton whose sign is reversed in going from $x^{+}<0$ to $x^{+}>0$. Since in the Einstein frame the dilaton acts as a scale factor, this sign reversal implies the transition from a big bang to a big crunch. The mathematical consistency of this gluing procedure $[27,28]$ requires the existence of a nontrivial NeveuSchwarz (NS) $B$-field supported only at the gluing junction. As we will show, this background can also be obtained as a solution to the IIA supergravity equations of motion.

\footnotetext{
${ }^{1}$ An everywhere vanishing answer was obtained in [7] although it is believed that this is due to calculating a time averaged version of the potential.

${ }^{2}$ As we will see, a particular sign for the dilaton corresponds to a choice between incoming or outgoing geodesics.
} 
For both the big bang and big crunch singularities, this solution reduces to two copies of the LLD. The new ingredient of this construction is the cosmological turning point, $x^{+}=0$, at which the theory initially appears to be singular. We study the supergravity description and find a background in terms of a regulating parameter, which once taken to zero leads back to the original solution. We will argue that the generic features of [1] are still valid and that, in both the far past and far future, one can construct a mildly modified version of the matrix string theory describing both null cosmological singularities. Once again the interpretation of the turning point is less transparent, but the physics appear to be captured by perturbative strings. Hence, it is still possible to write down a matrix string theory valid even at the turning point.

The rest of this note is organized as follows: In Sec. II we briefly review the lightlike linear dilaton background and the matrix big bang scenario of [1]. In Sec. III we analyze the situation when viewed as a Penrose limit of a nonextremal NS5 solution. We then go on to describe the big bang-big crunch scenario as a gluing procedure in Sec. IV. Section V is devoted to obtaining the same solution from 10D supergravity and providing a regularized version before we discuss the matrix string theory description in Sec. VI. We summarize and conclude in Sec. VII.

\section{REVIEW OF THE LIGHTLIKE LINEAR DILATON BACKGROUND}

The LLD background is a remarkably simple timedependent solution of type IIA string theory involving flat Minkowski space in coordinates $x^{\mu}=\left(x^{+}, x^{-}, \vec{x}\right)$, with $\vec{x}$ representing the eight remaining spacelike directions, and string frame metric

$$
d s_{10}^{2}=-2 d x^{+} d x^{-}+d \vec{x}^{2} .
$$

There is also a dilaton given by $\phi=-Q x^{+}$, where $Q$ is a constant. Flat space is still a string solution despite the presence of the lightlike linear dilaton, since the latter, as opposed to a spacelike or timelike one, makes no contribution to the conformal anomaly [29].

By expressing the solution in the Einstein frame, the metric is rescaled by a factor of $e^{-\phi / 2}$ giving

$$
d s_{E}^{2}=e^{Q x^{+} / 2} d s_{10}^{2} .
$$

Cosmological evolution takes place in this frame. If one interprets $x^{+}$as the time variable, space-time originates at a big bang as $x^{+} \rightarrow-\infty$ provided that $Q>0$.

It is not just that the metric vanishes as $x^{+} \rightarrow-\infty$; it is clear that the string coupling $g_{s}=e^{\phi}$ blows up in this limit and therefore we should really be thinking about M-theory rather than IIA strings. Looking at the M-theory up-lift

$$
d s_{11}^{2}=e^{2 Q x^{+} / 3} d s_{10}^{2}+e^{-4 Q x^{+} / 3}\left(d x^{10}\right)^{2},
$$

with $x^{10}$ the 11 th direction, it is not hard to verify that there are divergent components of the Riemann tensor and that the space-time is geodesically incomplete [1]. The behavior of the solution (2.2) is thus that of a cosmology with an initial big bang singularity in light-cone time.

In [1], Craps et al. then go on to resolve this singularity by going to a dual matrix model description in terms of a 2D super Yang-Mills theory. This can be understood either as the gauge theory defined on the cylinder with a timedependent coupling, or as it having a constant coupling but being defined on a time-dependent world sheet given by the forward quadrant of the Milne orbifold of 2D Minkowski space. In this description the matrix model is weakly coupled when the string theory is strongly coupled (i.e. near the big bang singularity), which renders it tractable. We will return to this in Sec. VI.

The constant $Q$, in principle arbitrary, should be taken to be positive if we are to interpret this solution as a big bang cosmology. By means of a boost $x^{+} \rightarrow \frac{x^{+}}{Q}$ and $x^{-} \rightarrow Q x^{-}$, we can always set the background to

$$
d s_{10}^{2}=-2 d x^{+} d x^{-}+d \vec{x}^{2}, \quad \phi=-x^{+}
$$

in string frame or

$$
d s_{E}^{2}=e^{x^{+} / 2}\left(-2 d x^{+} d x^{-}+d \vec{x}^{2}\right), \quad \phi=-x^{+}
$$

in Einstein frame, where we have a big bang singularity in the far past of light-cone time $x^{+}$. The string coupling, $g_{s}=e^{-x^{+}}$also diverges at the singularity.

\section{PENROSE LIMIT OF NONEXTREMAL NS5S AND LLD}

It has been shown [26] that the linear dilaton background can be understood as a Penrose limit along a radial null geodesic of the solution for $N$ coincident NS5-branes. Roughly speaking, the Penrose limit amounts to boosting to the speed of light while at the same time blowing up the neighborhood of a given geodesic to the whole space. Given that an observer falling freely into a black hole cannot distinguish between the existence (or nonexistence) of horizons, it is to be expected that in both the extremal and nonextremal NS5 cases the Penrose limit will yield the same space. Indeed one can check explicitly that this is the case, so for the sake of generality let us consider the near horizon limit of the nonextremal NS5-brane solution [30]. This is given by

$$
\begin{aligned}
d s^{2}= & -\left(1-\frac{r_{0}^{2}}{r^{2}}\right) d t^{2}+d \vec{y}^{2}+\frac{N l_{s}^{2}}{r^{2}}\left\{\frac{d r^{2}}{\left(1-\frac{r_{0}^{2}}{r^{2}}\right)}\right. \\
& \left.+r^{2}\left(\cos ^{2} \theta d \psi^{2}+d \theta^{2}+\sin ^{2} \theta d \varphi^{2}\right)\right\},
\end{aligned}
$$

where $\vec{y}$ corresponds to the five world volume coordinates of the NS5-branes, which are located at $r=0$. The geometry in $\{t, r\}$ is that of a $2 \mathrm{D}$ black hole whose horizon sits at $r=r_{0}$. There is also a nonzero dilaton given by 


$$
e^{2 \phi}=\frac{\tilde{g}_{s}^{2} N l_{s}^{2}}{r^{2}}
$$

where $\tilde{g}_{s}$ is the asymptotically weak string coupling constant, and an NS 3-form field strength given by

$$
H_{(3)}=N l_{s}^{2} \sin \theta \cos \theta d \psi \wedge d \theta \wedge d \varphi .
$$

We will be interested in Penrose limits of this geometry along a purely radial null geodesic. We closely follow the conventions of [26] and therefore parametrize the geodesic as

$$
\left(\frac{d r}{d u}\right)^{2}=\frac{r^{2}}{N l_{s}^{2}}, \quad d t=\frac{d u}{\left(1-\frac{r_{0}^{2}}{r^{2}}\right)}+d v .
$$

The first expression has the globally defined solution

$$
r=\bar{r} \exp \left(\frac{ \pm u}{\sqrt{N l_{s}^{2}}}\right)
$$

where the choice of sign corresponds to the choice between an incoming or an outgoing geodesic. This follows from the fact that if we compute the light-cone time velocity $\frac{d r}{d u}$, this is either positive, and thus an outgoing geodesic, or negative and thus an incoming geodesic. The parameter $\bar{r}$ is an integration constant which sets the overall scale of the space.

We now introduce the following change of variables:

$$
\begin{aligned}
& u=x^{+} \sqrt{N l_{s}^{2}}, \quad v=\frac{x^{-}}{\sqrt{N l_{s}^{2}}}, \\
& \theta=\frac{z}{\sqrt{N l_{s}^{2}}}, \quad \psi=\frac{w}{\sqrt{N l_{s}^{2}}},
\end{aligned}
$$

and after taking $N \rightarrow \infty$ one can easily see that the metric reduces to flat space in light-cone coordinates while the 3form field vanishes in this limit. Finally, the dilaton becomes

$$
e^{\phi}=\frac{\tilde{g}_{s} \sqrt{N l_{s}^{2}}}{\bar{r}} e^{\mp x^{+}}
$$

where we have implicitly assumed that the factor $\frac{\tilde{s}_{s} \sqrt{N l_{s}^{2}}}{\bar{r}}$ is positive and we will also require it to be finite and small. This is a reasonable assumption to make, ${ }^{3}$ implemented by fine-tuning the asymptotic string coupling $\tilde{g}_{s}$ to be weak and setting the integration parameter $\bar{r}$ to scale with $N$.

From (3.5) we see that there are two possible solutions for the radial geodesic corresponding to a particular choice of sign. Let us choose the positive solution for definiteness which gives $r=\bar{r} e^{x^{+}}$. After taking the Penrose limit [and absorbing the constant factor in Eq. (3.7)], we obtain

$$
d s^{2}=-2 d x^{+} d x^{-}+d \vec{x}^{2}, \quad \phi=-x^{+},
$$

which is precisely the linear dilaton background that we

\footnotetext{
${ }^{3} \mathrm{We}$ will come back to this issue in Sec. VII.
}

found in (2.4) and (2.5) and was analyzed in [1]. ${ }^{4}$ From this point of view, the big bang singularity at $x^{+}=-\infty$ should be associated with $r=0$, where the geodesic along which we took the Penrose limit is hitting the NS5-branes. Had we chosen the opposite sign, the geodesic would have hit the branes at $x^{+}=\infty$, which from this point of view should correspond to a big crunch singularity. Indeed, this picture can be verified by repeating the geodesic completeness analysis of Sec. II for a positive dilaton.

\section{THE BIG BRUNCH: GLUING THE BIG BANG TO THE BIG CRUNCH}

Given that we have a way to construct a big bang and a big crunch, it is natural to ask whether one can combine the two to obtain a closed cosmology. The obvious way of doing that would be to pick a section of each and glue them together to form a unique background involving both big bang and big crunch. Thus, let us choose as a "geodesic" for the Penrose limit

$$
r=\bar{r} e^{-x^{+}} \quad \text { if } x^{+}>0, \quad r=\bar{r} e^{x^{+}} \quad \text { if } x^{+}<0,
$$

which gives a dilaton

$$
\phi=x^{+} \quad \text { if } x^{+}>0, \quad \phi=-x^{+} \quad \text { if } x^{+}<0 .
$$

With this prescription we are gluing both geodesics at $r=$ $\bar{r}$. The background obtained in this way corresponds to following geodesics going out of the NS5-branes at $x^{+}=$ $-\infty$, reaching a maximum point $\bar{r}$ at $x^{+}=0$, and then coming back at $x^{+}=\infty$. Thus, it should correspond to evolution from a big bang to a big crunch. One could ask if the reverse situation is also possible, namely, an evolution from a big crunch to a big bang. As it will become clear later on, this possibility is not allowed in terms of this particular description because of the well-known positive energy theorems.

We have already seen that in the first patch the background is described by (3.8), while in the second it is again (3.8) but with the sign for the dilaton reversed. Because of their construction as a Penrose limit, we know that both geometries are solutions to the supergravity equations of motion. However, nothing guarantees that the same will also hold for the gluing point. To find out whether our background indeed satisfies the supergravity equations of motion, we first have to properly take care of the junction conditions. This setup could be thought of as a simple "cut-and-paste" problem, which is well known in gravity: In order to resolve it we must ensure that both the metric and the extrinsic curvature are continuous functions when crossing the junction surface. If this is not the case some extra matter must be supplied at the junction so that its stress-energy tensor compensates for the discontinuity in the extrinsic curvature.

\footnotetext{
${ }^{4}$ This alternative description and its relation to little string theory was also briefly touched on in that reference.
} 
This procedure typically starts by choosing a vector normal to the gluing surface and using it to compute the extrinsic curvature. In this case, however, we are gluing two copies of $\mathbb{R}^{8}$ at $x^{+}=0$. This is a lightlike surface, for which the normal vector declines into tangency and the usual definition of the extrinsic curvature is no longer valid. In turn, a slight variation of the algorithm was developed in [27] to accommodate for this fact. We will follow the latter prescription, i.e. look for the "transverse" (in a sense which will be described below) extrinsic curvature to the surface of gluing and, if the former is nonzero, add some extra fields whose stress-energy tensor will account for the discontinuity. For this we need to revert to the Einstein frame.

We wish to glue the following two patches,

$$
\begin{aligned}
& d s_{1}^{2}=e^{x^{+} / 2}\left(-2 d x^{+} d x^{-}+d \vec{x}^{2}\right) \text { for } x^{+}<0 \\
& d s_{2}^{2}=e^{-x^{+} / 2}\left(-2 d x^{+} d x^{-}+d \vec{x}^{2}\right) \text { for } x^{+}>0
\end{aligned}
$$

at $x^{+}=0$. In order to proceed further we will make use of the stress-energy tensor $\tau$ as defined in [28] for a null shell, namely

$$
8 \pi \tau_{\mu \nu}=-\left[k_{\mu \nu}\right]+\frac{1}{2} \gamma l_{\mu} l_{\nu}
$$

where

$$
\left[k_{\mu \nu}\right]=-\frac{l^{\rho}}{2}\left(\partial_{\mu} g_{\nu \rho}+\partial_{\nu} g_{\mu \rho}\right)
$$

is the "extrinsic" curvature, as explicitly defined for the null case, and

$$
\gamma l_{\mu} l_{\nu}=\left[g^{\rho \lambda} \gamma_{\rho \lambda}\right] l_{\mu} l_{\nu}
$$

The square braces stand for the following prescription:

$$
[F]=\lim _{x^{+} \rightarrow 0}\left(F_{x^{+}<0}-F_{x^{+}>0}\right),
$$

i.e. $[F]$ is the difference between the initial and final $F$ when crossing the junction. Note that this expression knows about the ordering of the backgrounds in the sense that we are evolving from $x^{+}<0$ to $x^{+}>0$. The vector $l_{\mu}$ defines the direction tangent to the null surface along which we are gluing. For the complete details on the construction we refer the interested reader to [28].

By describing the gluing surface as the condition $f\left(x^{+}\right)=0$, we obtain $l_{\mu}$ as

$$
l_{\mu}=\partial_{\mu} f .
$$

Given that $f$ is just a function of $x^{+}$, it is clear that the only nonzero entry for $l_{\mu}$ will be the one along $x^{+}$. Indeed, it is easy to see that the only nonzero entry for the stress-energy tensor at the junction is $\tau_{++}$. This turns out to be constant and has support on the gluing surface, i.e.

$$
8 \pi \tau_{++}=-4 \delta\left(x^{+}\right) .
$$

In order to have a supergravity solution valid at the junc- tion, we need to add some extra matter which will compensate for this difference. Motivated by the fact that our background comes from a certain limit of an NS5 geometry, where the only extra field is the NS $B$-field, we will absorb (4.9) through the introduction of such a field. Its stress-energy tensor, in a suitable normalization for comparison with (4.9), reads

$$
T_{++}=-\frac{1}{32 \pi} H_{+\mu \nu} H_{+}{ }^{\mu \nu} .
$$

At this point we will impose an extra condition, assuming for the moment ${ }^{5}$ that $H_{\mu \nu \rho} H^{\mu \nu \rho}=0$. This then leads to

$$
H_{+\mu \nu} H_{+}{ }^{\mu \nu}=16 \delta\left(x^{+}\right) \text {. }
$$

The background that we end up with in string frame then reads

$$
\begin{gathered}
d s^{2}=-2 d x^{+} d x^{-}+d \vec{x}^{2}, \quad \phi=\left|x^{+}\right|, \\
H_{+\mu \nu} H_{+}{ }^{\mu \nu}=16 \delta\left(x^{+}\right) .
\end{gathered}
$$

It is straightforward to see that, had we wanted to use the other ordering when gluing the geodesics, namely, the one which leads to a big crunch followed by a big bang, we would have obtained the opposite sign in (4.9). Therefore it would have been impossible to account for that through the stress-energy tensor of any field, for which the sign is fixed, or positive tension object. However, this could in principle be circumvented by the introduction of a new effective field theory at the singularity, where string theory is strongly coupled and it is expected that one will have the appearance of new light degrees of freedom [31,32], or by introducing negative tension objects such as O-planes in the spirit of [33].

\section{EXPLORING THE SUPERGRAVITY PICTURE}

We have so far obtained a background by gluing two geodesics along a null hypersurface, in the process of engineering a space-time incorporating both big bang and big crunch singularities. The question now pertains to the supergravity construction of such a solution. We begin with pure IIA supergravity containing a nonzero $B$-field while keeping a flat metric in string frame. We also have a lightlike dilaton which is now a generic function of $x^{+}$. The equations of motion are given by

$$
\begin{gathered}
\frac{1}{4}\left(\frac{1}{6} g_{\mu \nu} H^{2}-H_{\mu \alpha \beta} H_{\nu}{ }^{\alpha \beta}\right)+2 \partial_{\mu} \partial_{\nu} \phi-g_{\mu \nu} \partial^{2} \phi \\
=R_{\mu \nu}-\frac{1}{2} g_{\mu \nu} R, \\
4(\partial \phi)^{2}-4 \partial^{2} \phi+R+\frac{1}{12} H^{2}=0,
\end{gathered}
$$

\footnotetext{
${ }^{5}$ We will provide good evidence in support of this assumption in the following section.
} 


$$
\partial_{\lambda} H_{\mu \nu}^{\lambda}-2 \partial_{\lambda} \phi H_{\mu \nu}^{\lambda}=0 .
$$

Guided by the findings of the previous section, we will assume the minimal form for the NS 3-form field strength, i.e. the only nonzero components of $H$ will be those with $H_{+i j}=H_{+i j}\left(x^{+}\right)$, where $i$ and $j$ are indices labeling the 8 spatial coordinates. All the equations of motion are then trivially satisfied with the exception of

$$
H_{+i j} H_{+}{ }^{i j}=8 \partial_{+}^{2} \phi .
$$

This simplicity arises because of the index structure of $H$ and the functional dependence of $H$ and $\phi$ only on $x^{+}$. This means that $\partial^{2} \phi,(\partial \phi)^{2}$, and $H^{2}=H_{\mu \nu \rho} H^{\mu \nu \rho}$ are all identically zero, therefore justifying the assumption we made in the previous section a posteriori. The equation of motion for the $B$-field is satisfied for similar reasons. The only nontrivial terms come from the Einstein equations which reduce to (5.4) and show that the dilaton acceleration is actually a source for the $B$-field. Indeed, by assuming a simple linear dilaton solution we get $H=0$, which brings us back to the case in [1]. Conversely had we set the $B$-field to zero, we would have obtained a linear dilaton solution. Note that the acceleration of the dilaton is proportional to the square of $H_{+i j}$, and therefore explicitly non-negative.

Ultimately the background solutions consistent with the equations of motion are

$$
\begin{gathered}
d s^{2}=-2 d x^{+} d x^{-}+d \vec{x}^{2}, \quad \phi=\phi\left(x^{+}\right), \\
H_{+i j}=H_{+i j}\left(x^{+}\right),
\end{gathered}
$$

where the dilaton and the $B$-field are related as in (5.4). The 3 -form NS field-strength $H$ is given in terms of the 2-form potential as usual, $H=d B$. However, as we require spatial isotropy and homogeneity we will demand that $H_{+i j}=$ $\partial_{+} B_{i j}\left(x^{+}\right)$.

So far, we have constructed a set of supergravity solutions for a generic lightlike dilaton. Now, in very much the spirit of the background constructed with the cut-and-paste procedure, we will take ${ }^{6}$ the dilaton to be $\phi\left(x^{+}\right)=\left|x^{+}\right|$ since this has the requisite linear behavior in both domains. In this case

$$
H_{+i j} H_{+}{ }^{i j}=16 \delta\left(x^{+}\right) .
$$

The field $H_{+i j}^{2}$ is zero everywhere apart from $x^{+}=0$. This is precisely the cut-and-paste solution as obtained from the NS5-brane background (4.11), arising now in a more natural way within the supergravity context. To summarize, the explicit solution for our space-time is

$$
\begin{gathered}
d s^{2}=-2 d x^{+} d x^{-}+d \vec{x}^{2}, \quad \phi=\left|x^{+}\right|, \\
H_{+i j}^{2}=16 \delta\left(x^{+}\right) .
\end{gathered}
$$

\footnotetext{
${ }^{6}$ Once again we have absorbed the asymptotic value of the dilaton in this definition.
}

We would like to point out that the above falls under a class of backgrounds already considered in the literature [34-37]. However, in this note we concentrate on a particular example, namely, the one representing the big bangbig crunch cosmology.

\section{A. Supersymmetry considerations}

Since the time dependence of the problem has been introduced in the form of light-cone time, we anticipate that our background will preserve some fraction of supersymmetry. Here we will explicitly show this by looking at the vanishing of the supersymmetry variations relevant to the bosonic sector. These yield

$$
\begin{aligned}
\delta \psi_{\mu} & =\left(\partial_{\mu}-\frac{1}{8} \Gamma^{11} \Gamma^{\nu} \Gamma^{\rho} H_{\mu \nu \rho}\right) \epsilon, \\
\delta \lambda & =\left(\Gamma^{+} \partial_{+} \phi-\frac{1}{12} \Gamma^{11} \Gamma^{+} \Gamma^{i} \Gamma^{j} H_{+i j}\right) \epsilon .
\end{aligned}
$$

The Clifford algebra relation $\left\{\Gamma^{+}, \Gamma^{i}\right\}=0$ allows the variation of the gaugino to be rewritten as

$$
\left(\partial_{+} \phi-\frac{1}{12} \Gamma^{11} \Gamma^{i} \Gamma^{j} H_{+i j}\right) \times \Gamma^{+} \epsilon=0 .
$$

This holds, provided that $\epsilon$ is in the kernel of $\Gamma^{+}$. If we now examine the variation of the gravitino, since $H$ has no $H_{-\mu \nu}$ component, the only nontrivial equations are those for $\mu=+$ and $\mu=i$. For the latter we find that the equation is of the form

$$
\left(\partial_{i}+\frac{1}{8} \Gamma^{11} \Gamma^{j} H_{+i j} \Gamma^{+}\right) \epsilon=0,
$$

which is satisfied by decomposing $\epsilon=f\left(x^{+}\right) \epsilon_{0}$ with $\epsilon_{0}$ a constant spinor. We are thus left with the equation for $\mu=$ + . This reads

$$
\left(\partial_{+} f-\frac{1}{8} \Gamma^{11} \Gamma^{i} \Gamma^{j} H_{+i j} f\right) \epsilon_{0}=0 .
$$

The solution is then given by

$$
f=e^{(1 / 8) \Gamma^{11} \Gamma^{i} \Gamma^{j} B_{i j}} .
$$

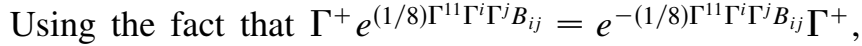
the condition $\Gamma^{+} \epsilon=0$ gets translated into $\Gamma^{+} \epsilon_{0}=0$ and we can thus conclude that the solution preserves 16 supersymmetries, i.e. the same amount as in the linear dilaton case. We therefore have a family of $\frac{1}{2}$-BPS backgrounds with metric, dilaton, and $B$-field as given by (5.5), with the additional constraint (5.4).

\section{B. Regularizing the solution}

The supergravity background just constructed exhibits a sharp localization at the turning point due to the delta function support for the $B$-field. In case this is of concern to the reader, we can smooth out this singular behavior by constructing a regularized version in terms of a scalar parameter $\epsilon$. We will then be able to take $\epsilon \rightarrow 0$ and 
recover the shell-like solution that we have thus far described. Let us consider the case where we choose the $B$ field to lie along two spatial directions such that $B_{12}$ is nonzero. This enables us to forget about the tensorial character of $H$ for the time being (though it would be easy to consider a more generic case) and allows us to define a regularized field strength,

$$
H_{+12}=\lim _{\epsilon \rightarrow 0} A \frac{\epsilon^{a}}{x^{+2}+\epsilon^{2}} .
$$

Here $A$ is a normalization factor and $a$ a suitable exponent to be fixed at a latter stage. The choice of this function is motivated by the fact that $H^{2}$ must be a $\delta$-function, the regularized version of which is a Lorentzian.

By using the equations of motion, we can obtain the corresponding dilaton. Since in the $\epsilon \rightarrow 0$ limit we should have an absolute value, this allows us to fix both $a$ and $A$. A short calculation, flat-space metric aside, reveals that the 3form field strength and dilaton are given by

$$
\begin{aligned}
\phi & =\frac{2}{\pi}\left(x^{+} \arctan \left(\frac{x^{+}}{\epsilon}\right)+\epsilon \log \epsilon\right), \\
H_{+12} & =\sqrt{\frac{32}{\pi}} \frac{\epsilon^{3 / 2}}{x^{+2}+\epsilon^{2}} .
\end{aligned}
$$

It can be shown analytically that the dilaton goes to $\phi=$ $\left|x^{+}\right|$in the limit $\epsilon \rightarrow 0$. In the case of $H$ the situation is more involved, however it can be proved that for a well behaved function $f\left(x^{+}\right)$(i.e. finite as $x^{+} \rightarrow \pm \infty$ ), as $\epsilon \rightarrow 0$

$$
\frac{1}{16} \int_{-\infty}^{\infty} d x^{+} H_{+12}^{2}\left(x^{+}\right) f\left(x^{+}\right)=f(0) .
$$

Therefore, the regularized solution exhibits the necessary $\delta$-function behavior and reduces to the original one in that limit.

At this point we have constructed a regularized version of the supergravity theory. In fact we can go one step further and argue that this family of backgrounds is also a string theory solution to all orders in $\sigma$-model perturbation theory. In [34] it was shown that a certain class of backgrounds involving a metric, dilaton, and NS $B$-field do not receive higher $\alpha^{\prime}$ corrections. These include fields of the form

$$
\begin{gathered}
d s^{2}=-d x^{+} d x^{-}+d \vec{x}^{2}+F\left(x^{+}, \vec{x}\right)\left(d x^{+}\right)^{2} \\
H_{\mu \nu \rho}=A_{i j}\left(x^{+}\right) l_{[\mu} \nabla_{\nu} x^{i} \nabla_{\rho]} x^{j} \quad \phi=\phi\left(x^{+}\right),
\end{gathered}
$$

where $l^{\mu}$ is a null Killing vector encapsulating the fact that the metric is independent of $x^{-}$. The above are then solutions to all orders in $\alpha^{\prime}$ if

$$
\partial^{2} F+\frac{1}{18} A_{i j} A^{i j}+2 \partial_{+}^{2} \phi=0 .
$$

Our solution has $F \equiv 0, \phi=\left|x^{+}\right|$, and $H_{+i j}=C A_{i j}$, where $C$ is a constant, together with a suitable rescaling of $x^{+}$and $x^{-}$to match the metrics. Up to some normaliza- tion, the condition (5.17) is thus the same as our equations of motion (5.6). Hence, the big bang-big crunch background that we have described belongs to the more general family studied in [34] and is a solution of string theory to all orders in $\alpha^{\prime}$. It is important to note that, because of the special $x^{+}$dependence of the fields, a similar statement also applies to the regularized version of the solution. Since we obtain the shell-like background through a path in parameter space which lies entirely inside this family of exact string solutions, we feel confident that it is indeed a good background for string propagation.

\section{A THEORY DESCRIBING THE BIG BANG-BIG CRUNCH}

Having ensured that we have obtained a fully consistent string background, we can start studying string propagation. Consider the (light-cone) time-dependent effective string coupling

$$
g_{s}\left(x^{+}\right)=g_{0} e^{\left|x^{+}\right|},
$$

where $g_{0} \equiv \frac{\tilde{g}_{s} \sqrt{N l_{s}^{2}}}{\bar{r}}$. From this we see that for $x^{+} \rightarrow \pm \infty$ the string coupling tends to infinity and so a perturbative expansion in string loops does not make sense.

Let us forget about the point $x^{+}=0$ for the moment. We are then left with strings in an LLD background, which become strongly coupled at the big bang or the big crunch in each respective patch. Thus, as proposed by Craps et al., we can conclude that away from $x^{+}=0$ the full dynamics will be captured by a matrix string theory. The latter is described by an action

$$
\begin{aligned}
S= & \frac{1}{2 \pi l_{s}^{2}} \int d^{2} \sigma \operatorname{Tr}\left(\frac{1}{2}\left(D_{\alpha} X^{i}\right)^{2}+\theta^{T} \gamma^{\alpha} D_{\alpha} \theta\right. \\
& +g_{s}^{2} l_{s}^{4} \pi^{2} F_{\alpha \beta}^{2}-\frac{1}{4 \pi^{2} g_{s}^{2} l_{s}^{4}}\left[X^{i}, X^{j}\right]^{2} \\
& \left.+\frac{1}{2 \pi g_{s} l_{s}^{2}} \theta^{T} \gamma_{i}\left[X^{i}, \theta\right]\right),
\end{aligned}
$$

with the periodic identification $\sigma \sim \sigma+2 \pi l_{s}$ and where the string coupling is given by (6.1). The Yang-Mills coupling is identified with the inverse product of the string length and the string coupling

$$
g_{\mathrm{YM}} \equiv \frac{1}{g_{s} l_{s}},
$$

and it is obvious that this gauge theory becomes weakly coupled when the string theory is strongly coupled and vice versa.

Proceeding in more detail along the lines of [1], we will once again assume a $B$-field whose only nonvanishing element is $B_{12}$. In the usual matrix string theory construction in flat space, one considers a lightlike compactification $x^{-} \sim x^{-}+2 \pi R$, which is accompanied by a small shift in $x^{+}$due to the Sen-Seiberg argument [38,39]. However, this 
is no longer a symmetry in this context due to the explicit dependence of the dilaton on that coordinate. We can get around this problem by considering the Lorentz transformation

$$
\begin{gathered}
x^{+}=e X^{+}, \quad x^{-}=\frac{X^{+}}{2 e}+\frac{X^{-}}{e}+\frac{X^{1}}{e}, \\
x^{9}=X^{+}+X^{1},
\end{gathered}
$$

in terms of which the original lightlike compactification $\left(x^{+}, x^{-}, x^{9}\right) \sim\left(x^{+}, x^{-}, x^{9}\right)+(0, R, e R)$ becomes just $X^{1} \sim$ $X^{1}+e R$, i.e. is only a usual spacelike compactification. Hence, we can first T-dualize along the $X^{1}$ direction and then perform an S-duality to end up with a system of coincident D1-branes wrapped on a spatial direction. The effective theory describing the latter is the sypersymmetric completion of the non-Abelian DBI action and it can be seen that it indeed reduces to its Yang-Mills truncation (6.2) in the limit where $e \rightarrow 0[1,25]$.

As it stands the above description is not valid at $x^{+}=0$, since at that point we also have the appearance of the NS $B$-field. However, because the latter is of the form $B=$ $B_{12}\left(x^{+}\right) d x^{1} \wedge d x^{2}$ it will remain unchanged under the Lorentz transformation. If we now perform the duality sequence, starting with the T-duality along $X^{1}$, we eventually arrive at

$$
B \rightarrow C^{(2)},
$$

i.e. we map the NS field into a Ramond-Ramond (RR) 2form potential. This naturally couples to the world volume theory of the D1-strings. Hence, the effect of $B$ will show up in the theory of IIA F1s as if it were a $C^{(2)}$ in the theory of IIB D1-strings, giving rise to a Chern-Simons term of the form

$$
S_{\mathrm{CS}}=\frac{1}{2 \pi l_{s}^{2}} \int \operatorname{Tr}(P[B]) .
$$

We conclude that, if we add this extra term $S_{\mathrm{CS}}$ to Eq. (6.2), we will obtain an action valid for all light-cone time, including $x^{+}=0$, the bosonic sector of which will be described by

$$
\begin{aligned}
S= & \frac{1}{2 \pi l_{s}^{2}} \int d^{2} \sigma \operatorname{Tr}\left(\frac{1}{2}\left(D_{a} X^{i}\right)^{2}+g_{s}^{2} l_{s}^{4} \pi^{2} F_{a b}^{2}\right. \\
& \left.-\frac{1}{4 \pi^{2} g_{s}^{2} l_{s}^{4}}\left[X^{i}, X^{j}\right]^{2}+D_{a} X^{1} D_{b} X^{2} \epsilon^{a b} B_{12}\right) .
\end{aligned}
$$

However, in order to be confident about the validity of our approach we should work with the regularized version of the background and perform the duality sequence in the explicit presence of the regulator. After going to the Lorentz transformed coordinates adapted to the spatial compactification, one can check that the background in string frame is

$$
d s^{2}=r e^{-\Delta}\left(-2 d X^{+} d X^{-}+\left(d X^{9}\right)^{2}+d \vec{x}^{2}\right),
$$

where

$$
\Delta=\frac{2}{\pi}\left(e X^{+} \arctan \left(\frac{e X^{+}}{\epsilon}\right)+\epsilon \log \epsilon\right) .
$$

The dilaton becomes

$$
\phi=-\Delta+\log \hat{r},
$$

where the parameter $\hat{r}$ is related to the radius of the original lightlike compactification as

$$
\hat{r}=\frac{e R}{2 \pi l_{s}} .
$$

Additionally the 3-form potential, which has become RR because of the S-duality, is given by

$$
F_{+12}=\sqrt{\frac{32}{\pi}} \frac{\epsilon^{3 / 2}}{e^{2}\left(X^{+}\right)^{2}+\epsilon^{2}} .
$$

We can now consider the theory of D1-strings. Following [1], we will parametrize the action by choosing the following gauge:

$$
X^{9}=\frac{\sigma}{r}, \quad X^{+}=\frac{\tau}{r \sqrt{2}}, \quad X^{-}=\frac{\tau}{r \sqrt{2}}+\sqrt{2} y .
$$

Then, starting with the effective action for a single D1brane we can plug in the ansatz and expand up to quadratic order in the fields. Once we take the gauge choice into account the action reduces to

$$
\begin{aligned}
S= & \frac{1}{4 \pi l_{s}^{2}} \int d^{2} \sigma\left[\left(\partial_{\tau} \vec{x}\right)^{2}+\left(\partial_{\tau} y\right)^{2}-\left(\partial_{\sigma} \vec{x}\right)^{2}-\left(\partial_{\sigma} y\right)^{2}\right. \\
& \left.+\epsilon^{a b} \partial_{a} x^{1} \partial_{b} x^{2} B_{12}\right] .
\end{aligned}
$$

This is the Abelian version of (6.7) but with $B$ replaced by its regularized counterpart. At the non-Abelian level, the action one would get would be nothing but (6.7) with the appropriately regularized fields. At this stage we can safely take the limit $\epsilon \rightarrow 0$ to exactly recover (6.7).

Given that the behavior of our action mimics that of [1], it will be valid at both the big bang and big crunch singularities. The main difference of our description lies in the introduction of the $B$-field at $x^{+}=0$. Around that point the string coupling constant is $\sim g_{0}$. By tuning this to be small enough, the commutator term in (6.7) is forced to vanish leading to the usual Green-Schwarz superstring in the presence of an NS $B$-field.

\section{CONCLUSIONS AND OUTLOOK}

In this note we have extended the scenario presented in [1] to include a cosmological evolution from a big bang to a big crunch. We were able to do so by appropriately gluing together two copies of the LLD background. The gluing procedure forced us to introduce an extra NS $B$-field which was supported only at the turning point, $x^{+}=0$. This particular solution was also seen as a certain representative of a class of supergravity solutions involving the metric tensor, $B$-field, and dilaton, which is a family of exact string backgrounds. Since the former was also obtained 
as a limit of a regularized solution belonging to the same class, we feel confident about its validity despite the $\delta$-function support for $B$. After obtaining the background involving the evolution from a big bang to a big crunch, we then proceeded along the lines of [1] to construct a matrix string theory description of the physics. This was shown to be valid at the big bang, the big crunch, and also at the turning point.

Despite the above results, the physical interpretation of the turning point is still quite unclear. This is closely related to the somewhat exotic character of the NS $B$-field whose field-strength satisfies $d H=0$ and $d * H=$ 0 . However, we argued that the turning point does indeed admit a perturbative description: In terms of the NS5 picture, we ought to ensure that

$$
g_{0}=\frac{\tilde{g}_{s} \sqrt{N l_{s}^{2}}}{\bar{r}} \ll 1,
$$

where $\tilde{g}_{s}$ is the usual asymptotic value for the string coupling of the original NS5s, which is naturally taken to be small so that perturbative strings are well defined. Moreover, it is easy to see that the curvature of the NS5 background is given by

$$
\frac{1}{N}\left(1-\frac{4 N l_{s}^{2}}{6 r^{2}}\right)
$$

Since we are interested in the limit where $N \rightarrow \infty$, if we are to keep this small around $r \simeq \bar{r}$ we should require that

$$
\frac{N l_{s}^{2}}{\bar{r}^{2}} \ll 1
$$

This can be satisfied provided that $\bar{r}$ is large enough in string units, and follows from our requirement that $\bar{r}$ scales with $N$. Given that the solution also remains under control in the NS5-brane description, it could be possible to find a correspondence between our setup and the NS5 world volume theory [40] or little string theory [41-43]. In this context it would be nice to have a better understanding of the regularization, which could be related to a limiting procedure in the NS5 picture. However, even without referring to the NS5 interpretation, it seems clear that it is possible to go to a corner of the moduli space so that the theory is perturbative at the turning point. It would be interesting to perform a more detailed analysis of this point, in which the dual description in terms of the Milne orbifold world sheet of [1] could be of help. However, all these issues are beyond the scope of this note, and we will leave them open for future investigations.

\section{ACKNOWLEDGMENTS}

We would like to thank J. de Boer, B. Craps, B. Fiol, B. Janssen, Y. Lozano, K. Narayan, S. Ramgoolam, R. Roiban, K. Skenderis, S. Trivedi, M. A.Vazquez-Mozo, and $\mathrm{K}$. Zoubos for helpful comments and discussions. C.P. is grateful to the Centre for Research in String Theory at Queen Mary, University of London for generous hospitality and support while this work was in progress and to the people of India for supporting research in string theory. D. R-G. would like to thank Amsterdam University for kind hospitality while this work was in progress.
[1] B. Craps, S. Sethi, and E. P. Verlinde, J. High Energy Phys. 10 (2005) 005.

[2] T. Banks, W. Fischler, S. H. Shenker, and L. Susskind, Phys. Rev. D 55, 5112 (1997).

[3] L. Motl, hep-th/9701025.

[4] T. Banks and N. Seiberg, Nucl. Phys. B497, 41 (1997).

[5] R. Dijkgraaf, E. P. Verlinde, and H. L. Verlinde, Nucl. Phys. B500, 43 (1997).

[6] B. Craps, A. Rajaraman, and S. Sethi, Phys. Rev. D 73, 106005 (2006).

[7] M. Li and W. Song, J. High Energy Phys. 08 (2006) 089.

[8] S. R. Das and J. Michelson, Phys. Rev. D 72, 086005 (2005).

[9] S. R. Das and J. Michelson, Phys. Rev. D 73, 126006 (2006).

[10] D. Robbins and S. Sethi, J. High Energy Phys. 02 (2006) 052.

[11] E. J. Martinec, D. Robbins, and S. Sethi, J. High Energy Phys. 08 (2006) 025.

[12] J. H. She, J. High Energy Phys. 01 (2006) 002.

[13] M. Li and W. Song, J. High Energy Phys. 10 (2005) 073.
[14] M. Li, Phys. Lett. B 626, 202 (2005).

[15] B. Chen, Phys. Lett. B 632, 393 (2006).

[16] H. Z. Chen and B. Chen, Phys. Lett. B 638, 74 (2006).

[17] T. Ishino and N. Ohta, Phys. Lett. B 638, 105 (2006).

[18] C. S. Chu and P. M. Ho, J. High Energy Phys. 04 (2006) 013.

[19] F. L. Lin and W. Y. Wen, J. High Energy Phys. 05 (2006) 013.

[20] S. R. Das, J. Michelson, K. Narayan, and S.P. Trivedi, Phys. Rev. D 74, 026002 (2006).

[21] S. R. Das, J. Michelson, K. Narayan, and S. P. Trivedi, Phys. Rev. D 75, 026002 (2007).

[22] D. Bak, Phys. Rev. D 75, 026003 (2007).

[23] H. Kodama and N. Ohta, Prog. Theor. Phys. 116, 295 (2006).

[24] L. Cornalba and M. S. Costa, Fortschr. Phys. 52, 145 (2004).

[25] B. Craps, Classical Quantum Gravity 23, S849 (2006).

[26] V. E. Hubeny, M. Rangamani, and E. P. Verlinde, J. High Energy Phys. 10 (2002) 020.

[27] C. Barrabès and W. Israel, Phys. Rev. D 43, 1129 (1991). 
[28] C. Barrabes, Classical Quantum Gravity 6, 581 (1989).

[29] R. C. Myers, Phys. Lett. B 199, 371 (1987).

[30] G. T. Horowitz and A. Strominger, Nucl. Phys. B360, 197 (1991).

[31] J. Khoury, B. A. Ovrut, N. Seiberg, P. J. Steinhardt, and N. Turok, Phys. Rev. D 65, 086007 (2002).

[32] N. Seiberg, hep-th/0201039.

[33] L. Cornalba, M.S. Costa, and C. Kounnas, Nucl. Phys. B637, 378 (2002).

[34] G. T. Horowitz and A. R. Steif, Phys. Rev. Lett. 64, 260 (1990).

[35] T. Ishino, H. Kodama, and N. Ohta, Phys. Lett. B 631, 68 (2005).
[36] R. R. Nayak, K. L. Panigrahi, and S. Siwach, Phys. Lett. B 640, 214 (2006).

[37] N. Ohta and K. L. Panigrahi, Phys. Rev. D 74, 126003 (2006).

[38] A. Sen, Adv. Theor. Math. Phys. 2, 51 (1998).

[39] N. Seiberg, Phys. Rev. Lett. 79, 3577 (1997).

[40] I. A. Bandos, A. Nurmagambetov, and D. P. Sorokin, Nucl. Phys. B586, 315 (2000).

[41] M. Berkooz, M. Rozali, and N. Seiberg, Phys. Lett. B 408, 105 (1997).

[42] N. Seiberg, Phys. Lett. B 408, 98 (1997).

[43] O. Aharony, M. Berkooz, D. Kutasov, and N. Seiberg, J. High Energy Phys. 10 (1998) 004. 